\title{
¿POR QUÉ ES NECESARIA UNA DIDÁCTICA ESPECÍFICA PARA LA EDUCACIÓN SUPERIOR?
}

\section{WHY IS NECESSARY AN SPECIFIC DIDACTICS FOR HIGHER EDUCATION?}

\author{
Juan Carlos López Gutiérrez, PhD. \\ Doctor en Ciencias de la Educación (Cuba) \\ Docente de la Facultad de Educación, Ciencias y Tecnología de la Universidad Técnica \\ del Norte, Ecuador. \\ jclopez@utn.edu.ec \\ Isarelis Pérez Ones, MSc. \\ Máster en Ciencias, Tecnología y Sociedad (Cuba) \\ Estudiante del Doctorado en Políticas Públicas de la Facultad Latinoamericana de \\ Ciencias Sociales, Ecuador. \\ isarelis@gmail.com
}

\section{ARTÍCULO DE REFLEXIÓN}

Recibido: 22 de noviembre de 2017

Aceptado: 30 de diciembre de 2017

\section{RESUMEN}

La educación superior tiene sus propias características, por tanto, requiere una didáctica específica. Esta debe posibilitar el aprendizaje de alumnos que poseen conocimientos y experiencias previas, motivaciones y expectativas diversas, respecto a su proyecto personal y profesional. Sin embargo, abunda en los sistemas de educación superior, modelos educativos sólidos en términos de componentes y recursos, pero carentes fundamentaciones. El objetivo del presente trabajo es señalar el fundamento epistemológico de la didáctica universitaria. Para su realización se utilizó como técnica el análisis documental. Los resultados expresan el desarrollo de una didáctica universitaria, 
que responde a la evolución de la didáctica específica, y se sustenta en estudios con un fundamento epistemológico.

Palabras clave: educación superior, didáctica, didáctica específica, didáctica universitaria, análisis documental.

\section{ABSTRACT}

Higher education has its own characteristics; therefore it requires an specific didactic. The university didactic should be enabling to learn of students who possess previous knowledge and experiences, motivations and diverse expectations, regarding their personal and professional project. However, it abounds in higher education systems, solid educational models in terms of components and resources, but lacking foundations. The objective of this paper is to point out the epistemological foundation of university didactics. For its realization, the documentary analysis was used as a technique. The results express the development of a university didactic, which responds to the evolution of specific didactics, and is based on studies with an epistemological foundation.

Keywords: higher education, didactics, specific didactics, specific university didactics, documentary analysis.

\section{INTRODUCCIÓN}

Los cambios en los ámbitos humanos, científicos y económicos, que inciden y presionan cada vez más a las universidades. Estas instituciones se han visto en poco tiempo literalmente sacudidas (Moreno, 2011). Ello conlleva reformas que van desde la concepción de la organización institucional, académica, investigativa, hasta los procesos de vinculación con el objetivo de que las Instituciones de Educación Superior (IES) sean pertinentes, o sea que atienda las necesidades globales, nacionales y locales del desarrollo.

Numerosos autores han dedicado atención a este tema. Malagón (2006) considera que el modelo clásico de educación superior hizo crisis como resultado de los escenarios que configuran el nuevo orden mundial y regional. Otra mirada al tema acerca el debate al modelo académico y de ciencia que responde a la visión unívoca de la cultura occidental y por tanto a una forma unidireccional de pensamiento (Rivadeneira, 2013). Para abordar la 
didáctica universitaria entonces, es básico considerar los cambios de la sociedad contemporánea.

Díaz (2005) plantea que una de las razones que el proceso de Convergencia de la Unión Europea consideró fundamental para promover la renovación del sistema de enseñanza aprendizaje dominante en la educación superior, tiene su origen en la nueva organización social que preside actualmente la vida comunitaria de todas las sociedades avanzadas y que se conoce como sociedad del conocimiento. Ello se distingue por la aceleración de la innovación científica y tecnológica, así como por la rapidez de los flujos de información en una nueva dimensión del espacio y del tiempo (Ginés, 2004).

Hace treinta años ya, se planteaba que la pertinencia de lo que se podría llamar el enfoque clásico de la educación superior estaba en crisis en todas partes. Resulta sorprendente observar cómo se está erosionando la hasta hace no mucho tiempo indiscutida certeza en torno a la importancia intrínseca de las titulaciones, los conocimientos y la acción sociocultural generados por la educación superior (Sutz, 1997).

\section{REVISIÓN TEÓRICA}

\section{El componente didáctico}

Uno de los componentes que permite que la enseñanza superior pueda lograr su cometido es la Didáctica. En el mundo educacional una polémica continua gira alrededor de la Didáctica. El debate se ha centrado en sus orígenes, su condición de ciencia, su objeto de estudio, sus categorías, leyes, principios, modelos, evolución, su carácter general o disciplinar y en su relación con la Pedagogía y con las metodologías. La Didáctica es una respuesta a la necesidad de encontrar un equilibrio que armonice la relación entre las maneras de enseñar de los educadores y el aprendizaje de sus discípulos; una contradicción todavía por resolver (Abreu, Gallegos, Jácome y Martínez, 2017).

El término didáctica se refiere al arte de enseñar o instruir, es la disciplina pedagógica de carácter práctico y normativo que tiene por objeto específico la técnica de la enseñanza, esto es, la técnica de incentivar y orientar eficazmente a los alumnos en su aprendizaje. 
Además, se reconoce como un conjunto de técnicas destinadas a orientar la enseñanza mediante principios y procedimientos aplicables a todas las disciplinas, para que el aprendizaje de los mismos se lleve a cabo con mayor eficiencia (Díaz, 2016).

La didáctica involucra reflexiones metateóricas sobre sus propias corrientes, orientaciones teórico - disciplinares, modelos, conceptualizaciones, ejercicios de fundamentación y problemáticas; así como los aportes sobre la enseñanza como tal (teorías didácticas, modelos didácticos, investigaciones didácticas o sobre la enseñanza, estudios sobre aspectos específicos de la enseñanza (los contenidos, los saberes disciplinares, los métodos, etc.) ligadas también al ámbito profesional y de la praxis (puesta en práctica de la enseñanza orientada por el saber didáctico, planeación didáctica) (Díaz, 2005). Madrid y Mayorga (2010) consideran que la Didáctica es el campo de acción de numerosas investigaciones de proyecciones teóricas y prácticas, cuyos aportes han enriquecido el sistema de conocimientos y han determinado su carácter de ciencia en dos dimensiones, la teoría y la práctica.

Sin embargo, de forma general, a lo largo de los siglos la universidad como institución formadora, preocupada por la transmisión a las nuevas generaciones de los contenidos científicos y culturales, se ha desentendido de las modalidades que adopta la enseñanza, valorizando exclusivamente la imagen del docente universitario como experto en esos contenidos; el dominio del contenido se instala como garantía de la calidad independientemente de las formas en que ese contenido son puestas a disposición del aprendizaje de los estudiantes (Lucarelli, 2008).

La renovación (en las universidades) suele consistir generalmente en la creación de nuevas carreras, con nuevas materias o nuevas combinaciones y permutaciones de materias. Es decir, la innovación suele girar en torno a qué se enseña. De este modo se perpetúa otra inercia del pensamiento sobre cómo enseñar a nivel universitario (Lucarelli, 2008). La enseñanza en el nivel universitario es una práctica que requiere con urgencia ser asumida científicamente y con pertinencia social. Debe ser considerada como un campo de estudio que demanda mayores investigaciones, redefiniciones, validaciones y reconstrucciones teóricas para que como práctica pueda estar a tono con las exigencias de las transformaciones sociales, políticas, científicas y técnicas (Díaz, 1999). 
Esta situación se conjuga con un contexto histórico signado por la persistencia del pensamiento único, en sus distintas manifestaciones, y por una cultura centrada en la globalización de las formas y los valores. Según Santos (2000) esa configuración del pensamiento único corresponde en la actualidad a un momento transicional en el mundo del conocimiento: la transición entre un paradigma de las certezas que signó a la ciencia moderna, al paradigma de las incertidumbres que se está insinuando y del que tenemos sólo algunos indicios en nuestra cotidianeidad.

Se evidencia la necesidad que pretende resolver el presente trabajo, al señalar el fundamento epistemológico de la didáctica universitaria como objetivo del presente trabajo. Para atender este objetivo el trabajo presenta una estructura en la cual se parte de las exigencias del entorno a las universidades y el basamento del componente didáctico. Luego, con la utilización del análisis documental, se aborda la evolución de las didácticas específicas a la didáctica universitaria y los estudios con fundamento epistemológico, ambos como parte del análisis de los resultados.

\section{MATERIALES Y MÉTODOS}

En la realización del presente estudio se utilizó como técnica para obtener información de fuente secundaria el análisis documental como una forma de investigación técnica, un conjunto de operaciones intelectuales, que buscan describir y representar los documentos de forma unificada sistemática para facilitar su recuperación (Dulzaides y Molina, 2004). Comprende el procesamiento analítico - sintético que, a su vez, incluye la descripción bibliográfica y general de la fuente, la clasificación, indización, anotación, extracción, traducción y la confección de reseñas (García, 2002; Dulzaides y Molina, 2004).

Peña y Pirela (2007, p.56) comentan que: "el tema del análisis documental es complejo en esencia, por cuanto posee aristas de tipo lingüístico, psicológico-cognitivo, documental, social e informacional". Esta peculiar forma de acceder al conocimiento se ha dignificado y orientado a la búsqueda del pretendido rigor, con el que se ufana el homo academicus moderno. Este modelo busca, ante todo, posibilitar una investigación reflexiva - analítica (Botero, 2003). 


\section{ANÁLISIS DE RESULTADOS}

Los resultados obtenidos definen dos dimensiones para el análisis de la didáctica universitaria. Por una parte, el surgimiento de didácticas específicas en función de contenidos disciplinares, y por otra el fundamento epistemológico que subyace en la conformación de esta didáctica específica como disciplina en creciente consolidación.

\section{De las didácticas específicas a la didáctica universitaria}

El creciente interés por la Didáctica como ciencia no es casual. Está vinculado con la complejidad del mundo, con la necesidad de formación del talento humano para garantizar el desarrollo, con la aparición de nuevos problemas y conflictos y la persistencia de otros, con el insistente reclamo del derecho a la educación y la proximidad a la sociedad del conocimiento.

Un primer análisis ubica la perspectiva en el marco de un campo aún no consolidado en el contexto académico, el interés por la investigación didáctica es reciente y su producción no es numerosa, aunque se perfila en desarrollo creciente. En los eventos científicos ligados a la temática de la producción científica que toma como objeto la universidad, hay escasos informes que refieren a investigaciones sobre las prácticas del aula universitaria; si bien hubo una etapa en que esos trabajos productos de reflexiones de corte más filosófico que didáctico, aún en la actualidad muchos de ellos son más relatos e experiencias alternativas a las tradicionales que reflexiones sistemáticas acerca de la práctica (Lucarelli, 2008).

Moreno (2011) plantea que durante mucho tiempo se ha hecho referencia a la didáctica general a secas y más recientemente se distinguen distintos tipos de didácticas en función de los contenidos disciplinares a los que atienden, son las denominadas didácticas específicas, también registrada como didácticas especiales (Díaz, 2001; Grisales - Franco, 2012).

Tovar-Gálvez (2008) argumenta la existencia de una Didáctica General sustentada en las teorías del aprendizaje y que por ende se enfoca en los procesos cognitivos del sujeto y en lograr su desarrollo a través de estrategias de aprendizaje. Con el transcurso del tiempo, el 
ámbito de la didáctica general ha sufrido profundas transformaciones, debido al surgimiento de nuevas formas de conocimiento en educación, a los importantes avances de la investigación educativa, a la aparición de teorías psicológicas del aprendizaje, a la forma de concebir la inteligencia humana, entre otras (Moreno, 2011).

La existencia de didácticas específicas, cuyos soportes principales están en la historia y en la epistemología específicas de las disciplinas, buscan establecer estrategias de enseñanza de los contenidos disciplinares (indistintamente del modelo epistémico). Referirse a la didáctica específica es ubicarse en la asunción que las disciplinas, campos de conocimiento o formas de saber, tienen naturaleza, estructuras y dinámicas particulares; aspecto que se puede argumentar a través de los estudios históricos y epistemológicos de cada disciplina (Tovar - Gálvez y García, 2012).

Joshua y Dupin (1993), autores del primer manual universitario de didáctica de las ciencias, aportan un conjunto de reflexiones que sirven de marco justificativo para expresar el propio desenvolvimiento de la didáctica universitaria con la premisa de que la disciplina ha ido madurando poco a poco durante los últimos años, existiendo una opinión más o menos generalizada acerca de la creciente consolidación de la misma como cuerpo teórico y como comunidad académica (Gil-Pérez et al., 2000).

Se alude a un espacio disciplinar que se va generando, desdibujado en la actualidad, con referentes de prácticas cuyo trasfondo es expresión de un marco teórico conceptual propio de una didáctica universitaria. Conviene aclarar que en la actualidad esta es entendida como una disciplina específica dentro del campo didáctico, y como tal orientada a analizar el proceso de enseñanza que un docente o un equipo docente organiza en relación con los aprendizajes de los estudiantes y en función de un contenido altamente especializado y orientado hacia la formación en una profesión (Lucarelli, 1998).

Es considerada entonces, como una disciplina emergente, donde se reconoce la existencia de un conjunto de personas guiadas por la misma problemática, sistematizando un grupo de resultados coherentes con la especificidad de la educación superior que muestran la existencia de la producción de manuales, compilaciones y diccionarios de didáctica, y la sanción de planes de estudio de postgrado. 
Se considera la didáctica universitaria como un campo de estudios especializados sobre los procesos de enseñanza y aprendizaje en la universidad (Zabalza, 2007). Desde estas clarificaciones, la didáctica universitaria debe entenderse como una didáctica específica, comprometida con la organización de las intencionalidades de la enseñanza en el aula universitaria, con el diseño de variedad de estrategias de enseñanza en función de la significatividad de los aprendizajes del futuro profesional. Estas intencionalidades abarcan su desarrollo personal, su potencial de inteligencia, la adquisición de competencias profesionales y el desarrollo de actitudes y valores en función de su participación crítica y creativa en el contexto socio - político (Díaz, 2001).

Un resumen de las aproximaciones al tema, ubica un primer grupo de definiciones que se apoyan en la concepción de universidad como institución de enseñanza o institución educativa, y declaran el término pedagogía universitaria (Grisales - Franco, 2012). Otro grupo de autores, declaran la didáctica universitaria (De la Herrán, 2001; Pereira da Silva, Valdés y Fernández, 2005; Zabalza, 2007; Grisales - Franco, 2012; Díaz, 2016). Todas ellas son expresiones propias de un discurso histórico, reconociendo la universidad como una institución destinada a la producción de conocimiento, en la cual la enseñanza y otras funciones o actividades tienen cometidos y sentidos derivados (Behares, 2011).

Los criterios más usuales que permiten responder la especificidad de la didáctica universitaria, asumiendo la propuesta de (Bolívar, 2005; Camilloni, 2008 y Grisales Franco, 2012), se recogen en la siguiente tabla:

Tabla 1. Criterios más usuales que permiten responder la especificidad de la didáctica universitaria.

\begin{tabular}{|c|l|l|}
\hline No. & \multicolumn{1}{|c|}{ Criterio } & $\begin{array}{c}\text { Respuesta asumiendo la especificidad } \\
\text { universitaria }\end{array}$ \\
\hline a) & $\begin{array}{l}\text { Según los distintos niveles del } \\
\text { sistema educativo }\end{array}$ & Sistema universitario \\
\hline b) & Según las edades de los alumnos & Jóvenes adultos y adultos \\
\hline
\end{tabular}




\begin{tabular}{|c|l|l|}
\hline c) & Según las disciplinas & $\begin{array}{l}\text { Por el nivel de profundidad y el objetivo de } \\
\text { generar un nuevo conocimiento en cada } \\
\text { disciplina }\end{array}$ \\
\hline d) & $\begin{array}{l}\text { Según el tipo de institución: } \\
\text { educación formal o educación no } \\
\text { formal, con subdivisiones según se } \\
\text { trate }\end{array}$ & Universidades. \\
\hline
\end{tabular}

Fuente: Elaborado por el autor según (Bolívar, 2005; Camilloni, 2008 y Grisales - Franco, 2012).

Para Grisales - Franco (2012) estas contribuciones en el contexto de la enseñanza superior, llevan a comprender que el concepto didáctica universitaria no se agota en ser la teoría y la práctica de la enseñanza superior, sino que avanza a considerarla como un proceso de naturaleza sistémica y sistemática donde se relacionan diferentes elementos como principios, objetivos, contenidos, métodos, formas y medios - que posibilitan el tratamiento didáctico de los conocimientos.

Los elementos anteriormente declarados, se apoyan en las leyes de la didáctica de la educación superior, las que permiten un acercamiento a la interacción entre contexto pertinencia - didáctica si se reconoce (Ortiz, 2009): - Relación medio social - proceso; Relación componentes internos del proceso. Ello nos permite coincidir con Grisales Franco (2012) al comprender el concepto didáctica universitaria como una didáctica específica que se circunscribe a los procesos de enseñanza en el nivel superior de la educación para la formación de profesionales e investigadores.

Estas especificaciones y énfasis en el proceso universitario, se configuran a partir de los niveles que asume la didáctica como estructura del campo de estudio (Peterssen, 2001; Runge, 2013):

- En el nivel de la teoría (investigación, reflexión, teorización): pensamiento referido a la praxis didáctica, en donde la teoría sería precisamente el resultado de tal pensar y contendría enunciados sistemáticos acerca de lo efectivo de la enseñanza o cómo 
podría o debería ser. Como teoría es un cuerpo teórico que estudia los problemas relativos a la enseñanza superior con miras a posibilitar el aprendizaje de los estudiantes.

- El nivel de la praxis/profesión: fenómenos y procesos experienciales del enseñar y el aprender. Metódicas, práctica reflexiva. Es una traducción que crea el docente universitario para comunicar el sentido de los conocimientos que están expresados en un lenguaje específico y determinado, con el fin de posibilitar el entendimiento y la comprensión de dichos conocimientos.

\section{Los estudios con fundamento epistemológico}

Los resultados revelan que la didáctica universitaria se fundamenta en las nuevas posturas epistemológicas sobre el conocimiento y su construcción, así como en los paradigmas socio - cognitivos sobre el aprendizaje.

La didáctica universitaria puede conceptuarse como una didáctica especial comprometida con lo significativo de los aprendizajes del futuro profesional, con su desarrollo personal y con el potencial de su inteligencia en función de las exigencias del contexto socio-político.

Investigaciones recientes estiman que los cambios observados a nivel de estudiantes, en comparación con el entorno social y cultural, alientan a repensar en la misión de la universidad y los métodos de enseñanza al mismo tiempo, lo que deja en claro la necesidad de declarar que la preocupación, las tareas y la función del profesor evolucionan en orden a la profunda mutación que la universidad está presenciando (Jomaa, Chihi, Sghair, Mami y Kpazai, 2017). Se plantea una transformación del sistema de educación superior que haga que el alumno y el proceso de aprendizaje estén más enfocados (Liebrechta et al., 2017).

Por otra parte, las relaciones de la didáctica de las ciencias con la psicología del aprendizaje también marcan sucesivas etapas de consolidación de la disciplina. Actualmente, el aprendizaje es visto como objeto de estudio en tanto que es una actividad entre otras de gestión de saberes científicos en el aula. Los modelos didácticos y los psicológicos son distinguibles entre sí por sus intereses teóricos y prácticos, y por la atención que prestan a 
los contenidos específicos. Además, la atención al aprendizaje ha permitido a la didáctica de las ciencias conectarse a, y en muchos casos fundirse con, los estudios de naturaleza cognitiva (neurociencia, inteligencia artificial, teoría de sistemas expertos).

Al respecto, para avanzar en su comprensión, corresponde puntualizar algunas pautas:

a) Existe consenso en reconocer que los conocimientos constituyen la variable más importante en el desarrollo científico y tecnológico y los puntos de referencias para las nuevas formas de organización social presentes y futuras.

b) La enseñanza universitaria es la más comprometida con la producción, divulgación y aplicación de los conocimientos.

c) La intencionalidad, métodos, medios, espacios y recursos que utiliza la universidad para trabajar el conocimiento, demostrará su vigencia histórica y su capacidad para reorientar sus opciones didácticas o trabajo en el aula en función del desarrollo de la ciencia y de las reconstrucciones socioculturales de la sociedad actual.

d) La enseñanza universitaria no es una práctica aislada y con objetivos inmediatos como parecen asumir una gran parte del profesorado.

e) La enseñanza universitaria es un proceso complejo que implica: un enseñante calificado; alumnos socialmente comprometidos y con inteligencias potenciales; aprendizajes como experiencias significativas en cada alumno; contenidos temáticos, de procedimientos y actitudes apropiados tanto para el desarrollo integral del futuro profesional como para el desarrollo científico y; la puesta en marcha de currículos contextuados y en permanente revisión.

En cuanto a la producción y generación de conocimiento tiene una gran influencia las teorías desarrolladas en torno a los nuevos modos de producción del conocimiento (Funtowicz y Ravetz, 2000; Carayannis, Barth y Campbell, 2012; Villalobos, Band, Torres y González, 2016). Se habla de un cambio en las modalidades y en la institucionalización de la investigación y la formación de especialistas. Básicamente la idea es que se está pasando de una orientación disciplinaria a una de contexto de aplicación (Gallegos, Berra, Benito y López, 2014) en que tanto el conocimiento como la formación se hibridan, cruzando enfoques, actores, intereses y espacios organizativos. Esto lleva a una pérdida de importancia relativa respecto de su nivel histórico de la educación superior en tanto deja de 
ser, como lo había sido, socio privilegiado -cuando no exclusivo- de la combinación investigación - formación (Marcano, 2002).

Las universidades tienen como materia prima la generación de nuevos conocimientos, de ahí mientras mayor sea su capacidad en este terreno, mayores serán sus fortalezas. Esto significa un gran lance que obliga a las instituciones de educación superior a replantear sus modelos de formación (Moreno, 2011); es el papel de la universidad como productor de conocimiento, así como en un entorno para la educación y la formación, basado en la investigación científica sólida y la experiencia reflexiva (Gougoulakis, 2017).

La ruptura epistemológica que promueve esta nueva forma de organización del conocimiento y los aprendizajes, exige innovadoras formas de integración de la ciencia y los saberes. Los cursos universitarios deben establecer entornos de aprendizaje realistas en forma de fábricas de aprendizaje, cuyo objetivo es permitir que los estudiantes actúen de forma autodeterminada y orientada a los objetivos en un entorno de producción global a la vez que promueven las competencias y habilidades relacionadas (Lanza, Minges, Stoll, Moser y Haefner, 2016).

Una presencia todavía tímida en el contexto latinoamericano ha sido la ecología de saberes. La misma ha reconocido un paradigma de la monocultura del saber y del rigor en la educación superior. Es decir, la idea de que el único saber riguroso es el saber científico, $\mathrm{y}$, por lo tanto, otros conocimientos no tienen la validez ni el rigor del conocimiento científico (De Souza, 2006). Se trata entonces de sustituir las monoculturas por las ecologías, donde se crea la posibilidad de que las experiencias ausentes se vuelvan presentes. Es la ecología de los saberes.

Una particularidad de estos tiempos es que esa didáctica universitaria debe romper la tradicional concepción de la academia de ser una amenaza para la preservación, reproducción y acceso a los conocimientos tradicionales y a los saberes ancestrales. Las propias estructuras sociales, la escuela, el instituto y la universidad han mantenido mecanismos de segregación y blanqueamiento de las culturas nacionales (Rivadeneira, 2013). Es que el diálogo de saberes no sólo se refiere a la incorporación de otros saberes a las aulas, sino a la posibilidad de interacción y dialéctica entre ciencias -las fácticas y las 
sociales-, en el mundo de las ideas y la experimentación, en la integralidad de la práctica académica y también en la investigación.

Esto equivale a realizar rupturas de modelos didácticos (que solo reorientan una práctica pedagógica basada en la instrumentación). Por consiguiente, es importante establecer nuevas formas de pensar y accionar en las universidades, espacios donde se dé el encuentro de culturas, el proceso introspectivo, la discusión socializada, el reconocimiento al pensamiento divergente, la diversidad metodológica y la comprensión de las situaciones, principios que describen a una didáctica universitaria que refleja una formación docente significativa (González, 2016).

\section{CONCLUSIONES}

El entorno y el acelerado desarrollo científico tecnológico exige un nuevo protagonismo de las universidades como instituciones comprometidas con la producción y distribución de conocimiento y la observancia de nuevas posturas epistemológicas.

El desarrollo alcanzado por las diferentes ramas que conforman las ciencias de la educación conllevan a profundas transformaciones en la didáctica general, ello ha generado el surgimiento de didácticas específicas en función de contenidos disciplinares, las cualidades de las materias y las características de los alumnos e instituciones.

Es necesaria una didáctica universitaria, pues esta responde a la evolución de una didáctica específica, y se sustenta en estudios con un fundamento epistemológico: responde a las especificidades de las diferentes materias - disciplinas que se imparten, a las formas del saber dictadas por la significancia de los perfiles profesionales de las carreras y, finalmente, por las dinámicas particulares de los procesos sustantivos: docencia, vinculación con la sociedad e investigación, con la peculiaridad de que deben ser observados y desarrollados integralmente. 


\section{REFERENCIAS BIBLIOGRÁFICAS}

Abreu, O., Gallegos, M., Jácome, J. y Martínez, R. (2017). La Didáctica: Epistemología y Definición en la Facultad de Ciencias Administrativas y Económicas de la Universidad Técnica del Norte del Ecuador. Formación universitaria. Vol. 10 (3)

Behares, L. E. (2011). Consideraciones sobre el sentido de las "pedagogías" y las "didácticas" universitarias, con especial referencia a la experiencia uruguaya. Educação, Santa María, v. 36, n. 3, pp. 337-350.

Bolívar, A. (2005). Conocimiento didáctico del contenido y didácticas específicas. Profesorado. Revista de currículum y formación del profesorado, 9 (2), pp. 1-39. Recuperado de: https://recyt.fecyt.es/index.php/profesorado/article/download/42681/24580

Botero, A. (2003). La metodología documental en la investigación jurídica: alcances y perspectivas2. Opinión Jurídica vol. 2, No. 4, pp. 109-116. Universidad de Medellín. Medellín.

Camilloni, A. (2008). Didáctica general y didácticas específicas. En A. de Camilloni, El saber didáctico pp. 23-39, Paidós, Buenos Aires.

Carayannis, E.; Barth, T. y Campbell, D. (2012). The Quintuple Helix innovation model: global warming as a challenge and driver for innovation. En: Journal of Innovation and Entrepreneurship, 1:2. Recuperado de: http://www.innovationentrepreneurship.com/content/1/1/2

De La Herrán, A. (2001). Didáctica universitaria. La cara dura de la Universidad. En: Revista Tendencias Pedagógicas. No. 6, pp. 11-38.

De Sousa Santos, B. (2006). Renovar la teoría crítica y reinventar la emancipación social (encuentros en Buenos Aires). CLACSO: Buenos Aires.

De Souza Santos, B. (2000). A critica da razao indolente. Contra o desperdicio da experiencia. Vol.1. Para um novo senso común. A ciencia, o dereito na transicao paradigmática. S. Paulo. Cortez.

Díaz, D. (2001). "Didáctica universitaria: una alternativa para transformar la enseñanza”. Acción Pedagógica, Vol. 10, Nos. 1 y 2, pp. 64-72.

Díaz, M. M. (2005). Cambio de paradigma metodológico en la Educación Superior. Exigencias que conlleva. Cuadernos de Integración Europea \#2, pp. 16-27. Recuperado

de: 
http://fido.palermo.edu/servicios dyc/encuentro2007/02 auspicios publicaciones/a ctas diseno/articulos pdf/A010.pdf

Díaz, V. (2016). Didáctica y prácticas en posgrado: una aproximación teórica. Revista UNIMAR, 34 (1), pp. 71-85. Nariño. Recuperado de: http://www.umariana.edu.co/ojseditorial/index.php/unimar/issue/viewFile/87/pdf 32

Dulzaides, M. E. y Molina, A. M. (2004). Análisis documental y de información: dos componentes de un mismo proceso. ACIMED Vol.12 No.2., La Habana

Funtowicz, S. O, y Ravetz, J. R. (2000). La ciencia posnormal: ciencia con la gente. Barcelona, España: Icaria Editorial.

Gallegos, M.; Berra, M.; Benito, E. y López, W. (2014). Las nuevas dinámicas del conocimiento científico y su impacto en la Psicología Latinoamericana. Psicoperspectivas, 13(3), 106-117. Recuperado en 28 de noviembre de 2017, de https://dx.doi.org/10.5027/psicoperspectivas-Vol13-Issue3fulltext-377

García, A. L. (2002). Tratamiento y análisis de la documentación. En: Vizcaya Alonso, D. (comp). Selección de lecturas: Fundamentos de la organización de la información. La Habana: Universidad de La Habana.

Gil-Pérez, D., Carrascosa, J. y Martínez-Terrades, S. (2000). Una disciplina emergente y un campo específico de investigación. En F.J. Perales y P. Cañal (Eds.), Didáctica de las ciencias experimentales. Teoría y práctica de la enseñanza de las ciencias (pp. 11-34). Alcoy: Marfil.

Ginés, J. (2004). La necesidad del cambio educativo para la sociedad del conocimiento. en: Revista Iberoamericana de Educación, mayo-agosto, número 035, pp. 13-37. Organización de Estados Iberoamericanos para la Educación, la Ciencia y la Cultura (OEI). Madrid.

González, A. (2016). Reorientación teórica de la didáctica universitaria, en: Didáctica, Aprendizaje y competencias: Un ejercicio de Investigación y conocimiento. Revista Educación Superior y Sociedad (ESS). IESALC, Caracas.

Gougoulakis, P. (2017). Educating Scientists. Philosophy and Practice of University Pedagogy. In: Academia. No 8, pp.35-75. Estocolmo.

Grisales-Franco, L.M. (2012). Aproximación histórica al concepto de didáctica universitaria. Educación y Educadores, vol. 15, N.2, mayo-agosto, pp. 203-218. Universidad de La Sabana. Cundinamarca. 
Jomaa Ben Hsouna, H. B.; Chihi, H.; Sghair, D.; Mami, M.; Kpazai, G. (2017). The Impact of Professional Experience on Teaching Conceptions in University -Case Studies from Tunisia. European Journal of Educational Sciences, EJES, September, Vol.4, No.3. ISSN 1857- 6036. http://dx.doi.org/10.19044/ejes.v4no3a7

Joshua, S. y Dupin, J. J. (1993): Introduction à la didactique des sciences et des mathématiques. Paris, Presses Universitaires de France.

Lanza, G., Minges, S., Stoll, J., Moser, E., Haefner B. (2016) Integrated and Modular Didactic and Methodological Concept for a Learning Factory. 6th CIRP Conference on Learning Factories, Procedia Manufacturing, Vol. 54, pp. 40-136.

Liebrechta, C., Hochdörffera, J., Trebera, S., Mosera, E., Erbachera, T., Gidionb, G., Lanzaa, G. (2017). Concept development for the verification of the didactic competence promotion for the Learning Factory on Global Production. Procedia Manufacturing, Vol. 9 pp. $315-322$.

Lucarelli, E. (2008). Asesoría pedagógica y cambio en la Universidad Profesorado. Revista de Currículum y Formación de Profesorado, vol. 12, núm. 1, 2008, pp. 1-14 Universidad de Granada, Granada, España. Recuperado de: http://www.redalyc.org/articulo.oa?id $=56717073009$

Madrid, D., Mayorga, M.J. (2010). ¿Didáctica General en y para Educación Social?. Puntos de encuentro desde la perspectiva del alumnado, Revista Educación Siglo XXI, 28(2), pp. 245-260. Recuperado de: http://revistas.um.es/educatio

Malagón Plata, L.A. (2006). La vinculación Universidad - Sociedad desde una perspectiva social. En: Educación y Educadores, Volumen 9, Número 2, pp. 79-93. Recuperado de: http://www.scielo.org.co/pdf/eded/v9n2/v9n2a07.pdf

Marcano, N. (2002). Pertinencia, compromiso social y currículo en las instituciones de educación superior. Encuentro educacional, 9(2). Recuperado en 28 de noviembre de 2017 , de http://www.produccioncientificaluz.org/index.php/encuentro/article/view/4335/0

Moreno Olivos, T. (2011). Didáctica de la Educación Superior: nuevos desafíos en el siglo XXI. En: Revista Perspectiva Educacional, Vol 50, $N^{\circ}$ 2, pp. 26-54. Universidad Autónoma Metropolitana Unidad-Cuajimalpa. México.

Ortiz, A. (2009) Pedagogía y aprendizaje profesional en la enseñanza superior. Ecoe Ediciones. Bogotá. 
Peña, T. y Pirela, J. (2007). La complejidad del análisis documental". En: Información, cultura y sociedad, (16), pp. 55-81. Recuperado de: http://www.redalyc.org/articulo.oa?id=263019682004

Pereira da Silva F.J.; Valdés Puentes, R. \& Fernández Aquino, O. (2005). Las investigaciones sobre formación de profesores en América Latina: un análisis de los estudios del estado del arte (1985-2003). En: Educação Unisinos, 9(3), pp. 221-230. Recuperado de: http://www.redalyc.org/articulo.oa?id=449644421005

Peterssen, W. (2001). Lehrbuch Allgemeine Didaktik. München: Oldenbourg Schulverlag.

Rivadeneira, M.I. (2013). El diálogo de saberes en los Estados Plurinacionales. Colección Cuadernos de Trabajo No. 1, SENESCYT, Quito.

Runge Peña, A.K. (2013). Didáctica: una introducción panorámica y comparada. Itinerario educativo. Año XXVIII, n. ${ }^{\circ}$ 62, Julio - diciembre, pp. 201-240.

Sutz, J. (1997). La universidad latinoamericana y su pertinencia: elementos para repensar el problema, en UNESCO- CRESAL: La educación superior en el siglo XXI. Visión de América Latina y el Caribe. Caracas: CRESAL-UNESCO, Tomo I.

Tovar-Gálvez, J. C., (2008). Modelo metacognitivo como integrador de estrategias de enseñanza y estrategias de aprendizaje de las ciencias, y su relación con las competencias. Revista Iberoamericana de Educación, 46(7). Recuperado de: http://www.rieoei.org/deloslectores/2161Tovarv2.pdf

Tovar-Gálvez, J.C. y García, G.A. (2012). Investigación en la práctica docente universitaria: obstáculos epistemológicos y alternativas desde la Didáctica General Constructivista. Educ. Pesqui., Vol. 38, No. 04, pp. 881-895.

Villalobos, C.; Band, A.; Torres, M. y González, S. (2016). Lógicas y modos de producción de conocimiento en política educativa: Análisis de la investigación producida en Chile (2000-2011). Revista iberoamericana de ciencia tecnología y sociedad, 11(33), 9-32. Recuperado en 28 de noviembre de 2017, de http://www.scielo.org.ar/scielo.php?script=sci arttext\&pid=S1850$00132016000300002 \& \operatorname{lng}=$ es\&tlng=es.

Zabalza Beraza, M. (2007). LA DIDÁCTICA UNIVERSITARIA. Bordón. Revista de $\left.\begin{array}{lllll}\text { Pedagogía, } & 59(2 & \text { y } & 3\end{array}\right) . \quad$ Recuperado de: https://recyt.fecyt.es/index.php/BORDON/article/view/36676 